\title{
Equi-Quantity, Equi-Calorie and Dose of Rice on Relative Glycemic and Insulinemic Response in Diabetic Patients
}

\author{
Kasturi Sen Ray, Neha V. Paharia \\ University Department of Food Science and Nutrition, S.N.D.T. Women's University, Juhu, Mumbai, India \\ Email: kasturisenray@gmail.com
}

Received November 2, 2013; revised December 2, 2013; accepted December 9, 2013

Copyright (C) 2013 Kasturi Sen Ray, Neha V. Paharia. This is an open access article distributed under the Creative Commons Attribution License, which permits unrestricted use, distribution, and reproduction in any medium, provided the original work is properly cited. In accordance of the Creative Commons Attribution License all Copyrights (C) 2013 are reserved for SCIRP and the owner of the intellectual property Kasturi Sen Ray, Neha V. Paharia. All Copyright (C) 2013 are guarded by law and by SCIRP as a guardian.

\begin{abstract}
Background and Aim: Diabetes mellitus is a chronic metabolic disorder with high blood sugar level. The postprandial glycemic impact of foods depends on the insulin status, which is deranged completely in a type 2 diabetic person. Dietary management of this group largely focuses on the low glycemic index (GI) food, based on equi-carbohydrate comparison, to keep the blood sugar level close to normal. But we consume whole food, along with other co-nutrients, moisture, fibre etc. The present study is aiming to assess the impact of main staple food rice with regards to Equi-Quantity, Equi-Calorie and Dose on relative glycemic and insulinemic response in diabetic patients as compared to normal group. Method: Blood samples of diabetic patients with stable blood sugar under medicinal treatment and paired normal patients $(n=6+6)$ were collected after an overnight fast and up to 2 hours post consumption of test and standard food on different occasions. Glucose and insulin levels were measured using glucometer (Abbott pharmaceuticals) and ECLIA method. Result: Equi-quantity of rice exerts a much lower glycemic and insulinemic response in comparison with bread in both normal and diabetic individuals and the response to rice does not show a proportional increase even when the quantities are doubled. Rice has higher moisture content which acts as energy diluent, decreasing the total starch in equivalent quantities. Equi-calorie (132 kcal) quantity comparison of rice $(100 \mathrm{~g})$ and bread $(50 \mathrm{~g})$ showed a much lower glycemic and insulinemic impact on rice in diabetic individuals, even though quantity is double and satiety level reaches earlier than low moisture food bread in equi-calorie quantity. The normal individuals, with normal insulin response can control the glycemic response to lower levels than those of diabetic subject. Conclusion: Rice having lower glycemic and insulinemic impact is a better suited food for diabetic individuals who already have a compromised insulin status.
\end{abstract}

Keywords: Diabetic Subjects; Rice; Glycemic Potency; Insulinemic Potency; Glycemic Bread Equivalent

\section{Introduction}

Diabetes mellitus is a chronic metabolic disorder affecting people across the globe. The number of people suffering from diabetes has recently a large increase, especially in the developing countries. Medication, diet and exercise are the cornerstone of diabetes management. Dietary management largely focuses on the quality of carbohydrate $(\mathrm{CHO})$ intake to keep the blood sugar level close to normal.

Presently, Glycemic Index (GI) value of food is used for selecting the food for their postprandial glycemic effect [1]. The major limitation with the use of GI is that the foods are assessed or compared on equi-carbohydrate bases. Food however is consumed as a whole with the presence of other co-components such as protein and fat along with moisture, fiber etc which individually and together influence the glycemic impact of any food. Also, in order to obtain equal amount of available $\mathrm{CHO}$, the quantity of food consumed will vary tremendously from one food to other and therefore comparison among the foods becomes difficult. This is a serious concern, especially for diabetic patients whose macronutrient metabolism is seriously affected due to deranged insulin status.

In order to overcome the limitations of GI, based on equi-carbohydrate basis, the term of Glycemic Index Foods $\left(\mathrm{GI}_{\text {Foods }}\right)$ was first introduced by J. A. Monro [2]. It is measured directly by the amount of reference food, bread, required to produce a glycemic response similar to the same amount of food. Thus while GI of foods is compared between foods on equi-carbohydrate basis, $\mathrm{GI}_{\mathrm{Foods}}$ 
are based on the glycemic impact of the entire food on equi-quantity basis. $\mathrm{GI}_{\mathrm{Foods}}$ can be used in exchange of food quantities with similar glycemic effect for management of sugars $[3,4]$.

The postprandial glycemic impact majorly depends on the insulin status of the person, especially in type 2 diabetic person. This insulin status is completely deranged, both in insulin resistant and insulin deficient type. Foods that trigger a lower insulin response may be beneficial in such cases. Therefore, Insulin Index $\mathrm{x}_{\text {food }}\left(\mathrm{II}_{\text {food }}\right)$ has also been considered for the characterization of specific carbohydrate rich foods [4].

Present study is focusing on equi-quantity $\left(\mathrm{GI}_{\mathrm{food}}\right.$, $\left.\mathrm{II}_{\text {food }}\right)$, dose response and equi-calorie $\left(\mathrm{GI}_{\text {Calorie, }}, \mathrm{II}_{\text {Calorie }}\right)$ based postprandial glycemic and insulinemic impact on type 2 diabetes persons who have an altered Glycemic and Insulinemic response to different foods. The response of diabetic person has been compared to the response from normal subject.

This may help us to make the effective dietary guideline for patients with diabetes.

\section{Materials and Method}

The research proposal was cleared through registered Ethics committee (Independent Ethics Committee, reg. no. 1433). The maturity onset diabetic subject at the age group of 45 - $65 \mathrm{yrs}$, without complication, with blood HbA1c level $<8 \%$, having comparatively stable sugar level with hypoglycemic medicine, along with age and BMI matched clinically healthy adults as normal group were enrolled after receiving informed written consent.

Selected Food Sample: The most commonly consumed staple Indian food rice (Surti Kolam variety), which is generally restricted in diabetic condition for its high GI value was selected as the experimental food. The rice, from the same batch, was prepared fresh every morning prior to the testing, following standardized procedure. White bread (Britannia Daily Fresh) was used as standard reference food.

Study Design: The same groups of subjects were tested for postprandial impact of equi-quantity, equi-calorie and dose response of rice and white bread on different occasion. After overnight fast, the subjects were given measured amount of food and asked to chew the given quantity of food thoroughly and finish within $10 \mathrm{~min}$. $100 \mathrm{ml}$ water was given with each serving.

Blood Analysis: Finger tip capillary blood was used for the estimation of blood glucose using a glucometer (Optium exceed, manufactured by Abbott pharmaceuticals) and venous blood was used for the estimation of insulin and $\mathrm{HbAlc}$ using ECLIA. The blood was collected in separate tubes for insulin and $\mathrm{HbAlc}$ (anticoagulant coated tube) estimation. Collection was made in the fasting state and at $30,60,90$ and 120 min post consumption of foods (standard and test). The incremental area under the curve (IAUC) for each food sample was calculated using the standard trapezoid rule.

Food Composition Analysis: The food samples were analyzed in triplicate for total, free reducing sugar and starch (Lane and Eynon method), Moisture (Vaccum oven method), Protein (Macro-kjeldhal method) and Fat (Soxhelet method) and Dietary Fiber (Enzymatic Digestion).

Statistical Analysis: Mean total glycemic and insulinemic IAUC and the peak glycemic values of test and standard food was compared using paired students $T$ test and was considered statistically significant at $p<0.05$ on two tailed testing. Standard deviation was calculated for the food composition values.

\section{Results}

\subsection{Relative Glycemic Impact on Equi-Quantity (GI $\mathrm{I}_{\text {Food }}$ Based Consumption}

Postprandial effect of food depends on its composition and the moisture content. Table 1 shows that total starch content in cooked rice is almost half and moisture content of rice is about double the amount as that of bread.

It is to be noted that fasting sugar level in normal subjects are about $6.2 \mathrm{mmols} / \mathrm{L}$, where as for diabetic subjects, it starts from around $8.7 \mathrm{mmols} / \mathrm{L}$, almost 1.4 times higher value for the present experimental group. As seen in Figure 1, rice peaks at half hour and starts falling towards the base line but does not touch the baseline within $2 \mathrm{hrs}(120 \mathrm{~min})$, which is a typical characteristic of diabetic patients. The glycemic impact of bread as standard food continues to increase and peaks at $60 \mathrm{~min}$. In normal subjects (Figure 1(b)) when equi-quantities of both bread and rice are fed, the blood sugars decline after 30

Table 1. Composition of standard and experimental food.

\begin{tabular}{|c|c|c|c|c|c|c|c|}
\hline \multirow[t]{2}{*}{ Food Product } & \multirow[t]{2}{*}{ Energy (kcal/100 g) } & \multicolumn{2}{|c|}{ Starch } & \multirow[t]{2}{*}{ Protein (g \%) } & \multirow[t]{2}{*}{ Fat $(\mathrm{g} \%)$} & \multirow[t]{2}{*}{ Moisture (g \%) } & \multirow[t]{2}{*}{ D. Fiber ( $\mathrm{g} \%)$} \\
\hline & & dwb g \% & wwb g \% & & & & \\
\hline W. Bread (Britannia) & 264 & $53.87 \pm 1.59$ & $33.97 \pm 0.69$ & $8.31 \pm 1.25$ & $1.71 \pm 0.95$ & $37.26 \pm 2.5$ & 4.04 \\
\hline Cooked Rice & 124 & $82.84 \pm 2.42$ & $20.47 \pm 0.38$ & $7.33 \pm 0.57$ & $1.35 \pm 0.91$ & $75.15 \pm 1.2$ & 3.61 \\
\hline
\end{tabular}

Mean \pm SD for each sample $(n=3)$. dwb — dry weight basis, wwb — wet weight basis. 


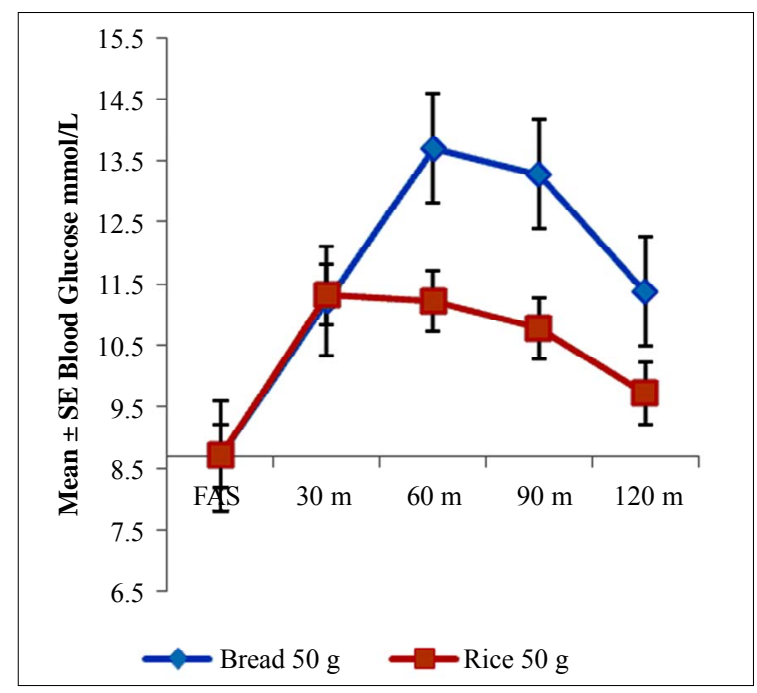

(a)

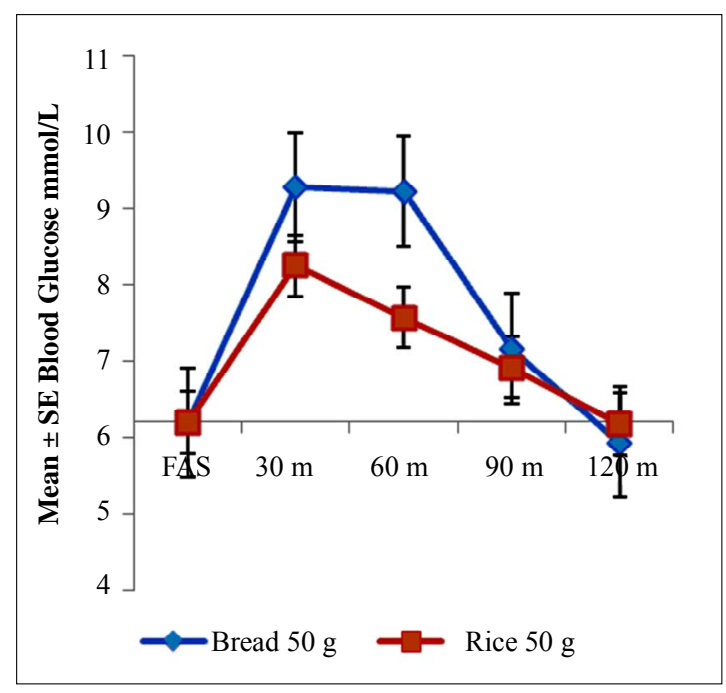

(b)

Figure 1. (a) Equi-quantity relative glycemic impact of rice on type II diabetic patients (n = 6); (b) Equi-quantity relative glycemic impact of rice in normal individuals $(n=6)$.

min and reaches baseline within $120 \mathrm{~min}$. These findings are similar to those observed in the study by Singhania and Senray [5]. The peak response obtained post consumption of bread is higher in both diabetic and normal subjects $(\mathrm{p}<0.05)$. Both the graphs (Figures 1(a) and (b)) clearly indicate that IAUC for both bread and rice is much higher in diabetic persons and AUC for rice is almost half of bread in both the groups. The area under the curve for rice is also seen to be significantly less than bread in both diabetic $(\mathrm{p}<0.05)$ and normal individuals $(\mathrm{p}<0.01)$.

\subsection{Relative Insulinemic Impact on Equi-Quantity Based Consumption}

Consumption of whole foods exerts varied insulinemic impact where macro-nutrient composition plays a major role. Foods which trigger lower insulin secretion may be beneficial in diabetic condition with reduced insulin synthesis or with insulin resistance. The present study also recorded the post prandial insulinemic impact exerted by bread and rice.

The normal fasting insulin levels varies from $2 \mathrm{mU} / \mathrm{L}$ to $25 \mathrm{mU} / \mathrm{L}$. As the range is very wide, fasting insulin level in two groups do not signify a lot but the difference in peak response and basal value is crucial. With similar glycemic load of rice, the normal group could generate about $32 \mathrm{mU}$ more insulin than diabetic group where difference is only $7 \mathrm{mU}$. But in response to equal amount of bread, made from wheat starch, the difference in basal and peak response is $20 \mathrm{mU}$ for diabetic group and 99 $\mathrm{mU}$ more for normal group. Therefore a normal individual with the capacity of producing sufficient functional insulin can maintain the blood sugar level efficiently.
In normal individuals (Figure 2(b)) the insulin level peaks at $30 \mathrm{~min}$ and decline steadily for rice. The higher insulin secretion in response to bread could be explained because of its high glycemic response along with higher protein and total starch content per serving (Table 1). The total area under the curve was also significantly lower for rice $(\mathrm{p}<0.05)$ as compared to bread.

In diabetic subjects the insulin levels for rice are seen to peak at $60 \mathrm{~min}$ post consumption and then gradually declines. However, it is noted that for rice, the insulin values are going closer to baseline at 120 min whereas, the insulinemic impact of bread continues to stay elevated. In normal group both sugar and insulin level goes back to fasting level.

\subsection{Dose Response}

In the present study $50 \mathrm{~g}$ and $100 \mathrm{~g}$ of rice was given to the same group of diabetic and normal subjects on different occasions and the responses were recorded. In diabetic individuals, the glycemic response of $100 \mathrm{~g}$ rice is not doubled but about 1.4 times of $50 \mathrm{~g}$ portion. In normal individuals of same age group, the glycemic and insulin response to $100 \mathrm{~g}$ rice is almost double that of 50 $\mathrm{g}$ portion (Figures 3 and $\mathbf{3 ( a )}$ ). These findings are similar with the findings of Sen Ray and Singhania [4] in normal young subjects.

The glycemic response of rice in comparison to bread has been calculated in terms of household measures as well as common serving measures for easier understanding (Table 2).

\subsection{Equi-Calorie}

Present study has noted the glycemic impact of equi- 


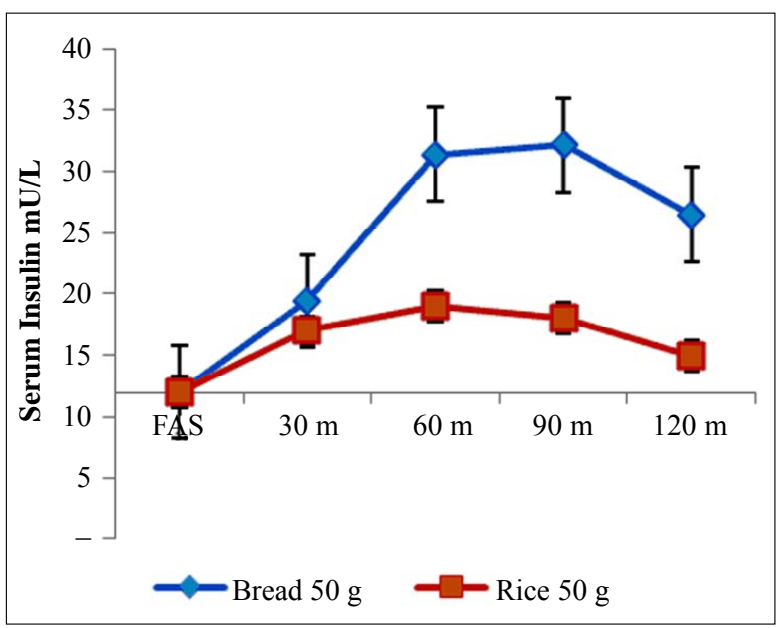

(a)

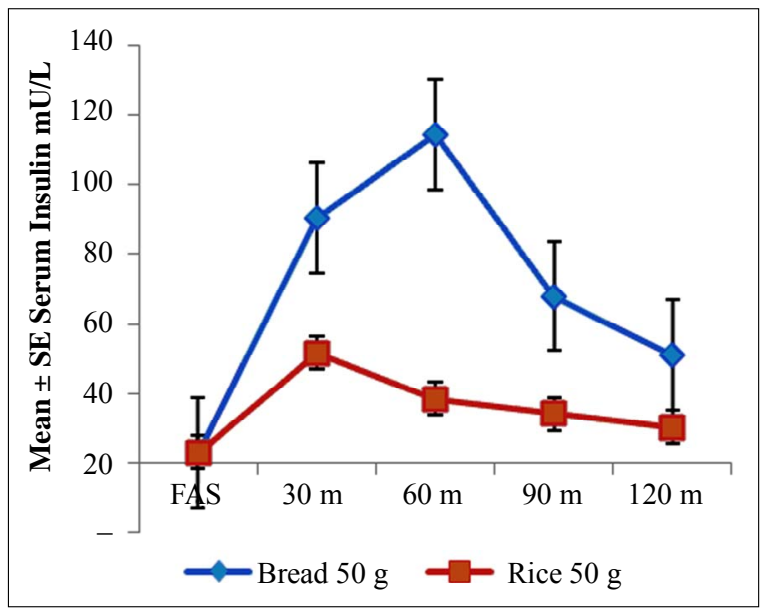

(b)

Figure 2. (a) Equi-quantity relative insulinemic impact of rice (50 g) in individuals with diabetes (type 2) (n = 6); (b) Equiquantity relative insulinemic impact of rice $(50 \mathrm{gm})$ in normal individuals $(\mathrm{n}=6)$.

Table 2. Glycemic bread equivalence (GBE) of rice in terms of household (HH) and common serving measure.

\begin{tabular}{|c|c|c|c|c|c|c|c|c|}
\hline \multirow{2}{*}{ Food product } & \multirow{2}{*}{$\begin{array}{l}\text { H.H. Measure of } \\
\text { one serving }\end{array}$} & \multicolumn{2}{|c|}{$\mathrm{GBE} / 50 \mathrm{~g}$ food } & \multirow{2}{*}{$\begin{array}{c}\text { Common Serving Measure } \\
\text { (CSM) }\end{array}$} & \multirow[t]{2}{*}{$\mathrm{Cal} / 50 \mathrm{~g}$} & \multicolumn{2}{|c|}{ Predicted GBE/CSM } & \multirow[t]{2}{*}{ Predicted cal/CSM } \\
\hline & & Normal & Diabetic & & & Normal & Diabetic & \\
\hline Bread & $11 / 2$ slices & 50 & 50 & 4 slices $(60 \mathrm{~g})$ & 132 & 60 & 60 & 158 \\
\hline Rice & $3 / 4^{\text {th }}$ cup & 27 & 30 & 1 cup $(130 \mathrm{~g})$ & 62 & 70 & 78 & 161 \\
\hline
\end{tabular}

calorific quantity of rice and bread for ready reference of exchange list for equicalorie-quantity. The amount of rice equivalent to calories provided by $50 \mathrm{~g}$ of bread (132 kcal), was calculated which corresponded to approximately $100 \mathrm{~g}$ of rice $(124 \mathrm{kcal})$.

The glycemic and insulin response in diabetic individual to equi-calorie-quantity of rice is lower than bread. In normal individuals the glycemic response to $100 \mathrm{gm}$ of rice is seen to be marginally high in comparison to 50 gm of bread (1.1 times). Despite this, the insulin requirement is found to be higher for half the quantity of bread $(50 \mathrm{~g})$ than rice $(100 \mathrm{~g})$ (Figure 4).

\section{Discussion}

Rice is a staple food for Indians and because of its high GI values, gets restricted for people with diabetes. In the present study we are comparing the glycemic impact of equi-quantity (50 gm) of rice and bread in diabetic subjects and compared the response with normal subjects.

Although GI value, based on equi-carbohydrate quantity, is high for both rice and bread, in practical condition, on equi-quantity basis, rice is not having very high glycemic impact due to its high moisture content and low carbohydrate content as shown in Figure 1.

The high glycemic impact of bread as compared to rice (Figures 1(a) and (b)) is due to the total starch content of bread which is 1.7 times higher (Table 1) than the same amount of cooked rice. Bread is low moisture food and contains more carbohydrate load in comparison to equal quantity of rice. The higher moisture content of rice $(75 \mathrm{~g}$ $\%$ ) causes a relative decrease in the total solid content including starch and acts as an energy diluent, resulting in a decrease in the total glycemic load of the meal.

When the glycemic responses of diabetic and normal individuals are compared it is seen that the glycemic response is 2.3 times higher for rice in diabetic subjects than normal and this could be due to the limited capacity of diabetic subjects with insulin functionality. Thus, it is obvious the glycemic AUC are much higher in diabetic patients than normal subjects (Table 3).

The important observation to be noted is that peak glycemic and insulinemic response of bread, made from wheat starch is high for both normal and diabetic person (Figures 1(a), 1(b), 2(a) and 2(b)). Glycemic response of wheat as compare to rice is almost same in normal and diabetic subjects (1.7 and 1.8 times) but Insulin response of wheat is as high as 3 times in normal subjects and double in diabetic subjects (Table 3) indicating that wheat has much higher insulinergic effect than rice.

The form in which the food is consumed also exerts an impact over the glycemic and insulinemic responses. Processing of food grains causes a disruption of their 
Table 3. Glycemic, insulinemic and GBE values for standard and test foods in normal and diabetic individuals.

\begin{tabular}{|c|c|c|c|c|c|c|c|c|}
\hline \multirow[b]{2}{*}{ Food } & \multicolumn{4}{|c|}{ Diabetic Individuals } & \multicolumn{4}{|c|}{ Normal Individuals } \\
\hline & Glycemic IAUC & Insulinemic IAUC & GBE & IBE & Glycemic IAUC & Insulinemic IAUC & GBE & IBE \\
\hline Bread (50 g) & 7470 & 1567.83 & 50 & 50 & 3582 & 6493.16 & 50 & 50 \\
\hline Rice $(50 \mathrm{~g})$ & 4455 & 729.83 & 30 & 23 & 1965 & 2199.65 & 27 & 17 \\
\hline Rice $(100 \mathrm{~g})$ & 6375 & 1243.68 & 43 & 40 & 3990 & 5646.85 & 56 & 43 \\
\hline
\end{tabular}

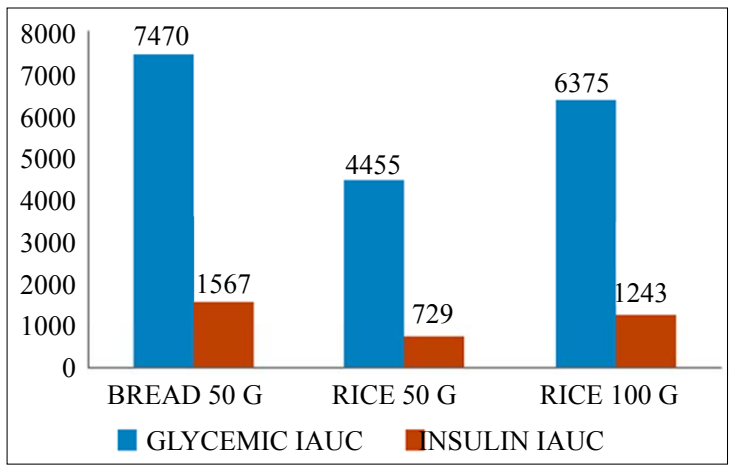

(a)

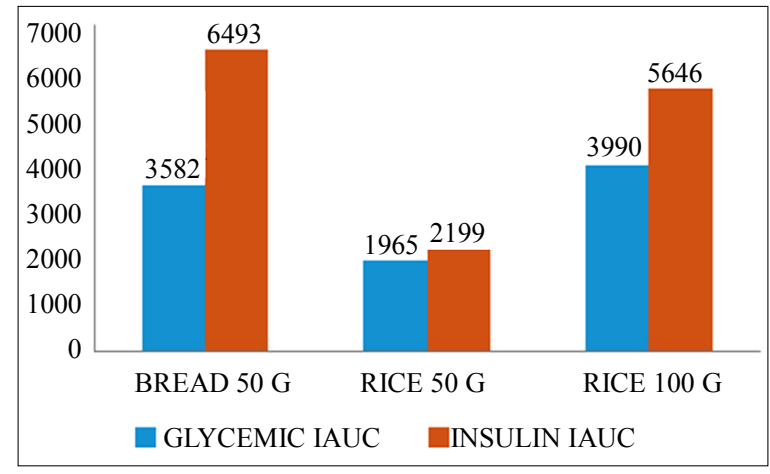

(b)

Figure 3. (a) Glycemic and insulinemic IAUC values in diabetic individuals (n = 6); (b) Glycemic and insulinemic IAUC values in normal individuals $(n=6)$.

natural structure and increases the digestibility of the starch present. Bread is made from refined wheat flour and this could be a probable reason for its high glycemic and insulinemic impact [6-8]. Further, consumption of cereals in their whole form, in this case rice, also help in decreasing the overall glycemic and insulin responses [9-11].

Besides the total starch content, the type of starch having different amylose: amylopectin ratio results different postprandial insulinergic and glycemic impact [12]. Rice has higher amylopectin content resulting faster absorption and utilization with lower requirement of insulin. Bread on the other hand leads to slow and sustained release of sugar causing an increased insulin secretion for its absorption [5]. The Figures 2(a) and (b) clearly indicate that the requirement of insulin for the utilization of rice is much lower than wheat starch granules in both diabetic and normal individuals. Higher requirement of insulin for wheat bread create further demand of insulin where diabetic patients are already compromised with their insulin status. The above findings also hold true the fact that the amount and rate of absorption of dietary starches $(\mathrm{CHO})$ are primary determinants of postprandial insulin [13].

Therefore it can be concluded that bread, made up of wheat, although not sweet in taste, still is detrimental for diabetic patients, having higher glycemic impact along with high demand for insulin. On the other hand rice is a better option as a carbohydrate source for diabetic person.
The lower insulinemic impact of rice may help in reducing the stress on the pancreas for its metabolism and this could be particularly beneficial in management of diabetes and people with insulin resistance $[4,5,9,14]$.

The portion size consumed by each individual is different and quantity of food consumed has an important contribution on postprandial glycemic impact, insulinemic impact and plays an important role in the dietary management of diabetic patient. Therefore dose response of rice has been assessed for its glycemic impact.

The graph (Figures 3(a) and (b)) is reemphasizing the fact that in normal subjects when insulin is sufficient, postprandial glycemic impact in terms of IAUC is lower as compared to diabetic person where insulin level is scarce; the postprandial sugar level is higher for both bread and rice.

The counseling of diabetic patients is completely based on controlling the postprandial blood sugar level close to normal value; therefore quality and quantity of $\mathrm{CHO}$ rich food are mostly controlled while calorific intake is maintained for $24 \mathrm{hrs}$. Figure $\mathbf{4}$ clearly indicates that the lower glycemic response in normal person as compared to diabetic group is because of sufficient insulin response. The insulin response in diabetic people is almost 4 times lower than normal people (Figure 4) and therefore the blood sugar levels continue to increase for bread. In normal individuals, the blood sugar levels for bread and rice are seen similar due to the availability of insulin. The graph again emphasizes that wheat starch is 


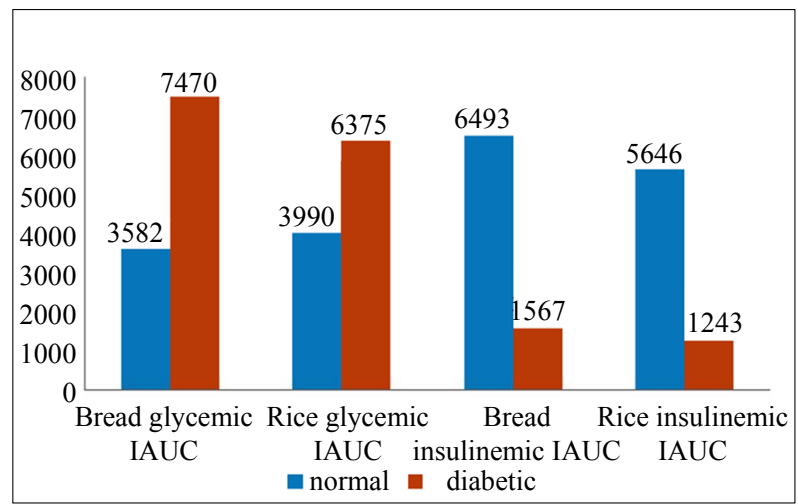

Figure 4. Equi-calorie glycemic and insulin AUC for bread $50 \mathrm{~g}$ (132 kcal) and rice $100 \mathrm{~g}$ (124 kcal) in normal and diabetic individuals $(n=6)$.

more insulinemic in nature as compared to rice starch (Figures 2(a) and (b)).

It is also seen that despite the total starch content of rice being marginally higher than that of bread $(20.47 \mathrm{~g}$ $\mathrm{v} / \mathrm{s}$ 16.98) on equi-calorie-quantity basis, it has a lower insulinemic impact. This proves that beside the quantity, quality of starch present in the food exerts a major impact on the post prandial glycemic and insulinemic responses.

Lower responses generated by twice the amount of rice has a high practical importance indicating that one can have larger portion size of rice as compare to bread, to satisfy the hunger. The portion size is generally reduced to control the postprandial blood sugar in diabetic patients and therefore cannot adhere to the dietary advice.

\section{Conclusions}

From the study, it is seen that impact of rice, based on equi-quantity and equi-calorie basis elicits a lower postprandial glycemic and insulinemic response as compared to bread in individuals with diabetes.

It is interesting to note that both these products belong to the same food group and have similar macronutrient composition. In this case, other various factors such as amylose-amylopectin content, particle size of the starch granule, cooking method, moisture content, form of food and the quantity of food consumed, play important contributory role in determining the overall glycemic impact of the food. Thus it is more important to classify foods based on their whole form and quantity consumed than those on single nutrient basis.

It is generally presumed that higher quantities of food will trigger a higher insulin response. Rice did not exert a proportional increase in the post prandial glycemic and insulinemic response when twice the quantity was consumed. The study also highlights the fact that the quality and quantity of whole food are important determinant of postprandial sugars.

In individuals with diabetes, prolonged increase in blood sugar levels is not desired on long term basis. It may lead to development of diabetes related complication. In such cases, the foods that tend to get digested rapidly along with a lower post prandial glycemic and insulin response are better suited. Rice, therefore can be considered as a suitable staple food in the dietary management of diabetes.

\section{Acknowledgements}

We thank the volunteers for their participation. Blood sample collection, HbA1c and Insulin was organized and analyzed by Clinical Research Services, SRL Limited, using double blind fold method. The Authors acknowledge the professional support.

\section{REFERENCES}

[1] D. J. A. Jenkins, T. M. S. Wolever, R. H. Taylor, H. Barker, H. Fielden, J. M. Baldwin, A. C. Bowling, H. C. Newman, A. L. Jenkins and D. V. Goff, "Glycemic Index of Foods: A Physiological Basis for Carbohydrate Exchange," American Journal of Clinical Nutrition, Vol. 34, No. 3, 1981, pp. 362-366.

[2] J. Monro, "Redefining the Glycemic Index for Dietary Management of Post Prandial Glycemia," Journal of Nutrition, Vol. 133, No. 12, 2003, pp. 4256-4258.

[3] J. A. Monro and M. Shaw, "Glycemic Impact, Glycemic Glucose Equivalents, Glycemic Index, and Glycemic Load: Definitions, Distinctions, and Implications," American Journal of Clinical Nutrition, Vol. 87, No. 1, 2008, pp. 237S-243S.

[4] K. SenRay and P. R. Singhania, "Glycemic and Insulinemic Responses to Carbohydrate Rich Whole Foods," Journal of Food Science and Technology, 2011.

[5] P. R. Singhania and K. SenRay, "Relative Glycemic and Insulinemic Impact of Rice and Rice Products," Nutrition and Food Science, Vol. 4, 2012, pp. 231-240.

[6] J. C. Brand, P. L. Nicholson, A. W. Thorburn and A. S. Truswell, "Food Processing and Glycemic Index," American Journal of Clinical Nutrition, Vol. 42, No. 6, 1985, pp. 1192-1196.

[7] A. E. Jarvi, B. S. Karlstrom, Y. E. Granfeldt, I. M. E. Bjorck, B. O. H. Vessby and N. G. L. Asp, "The Influence of Food Structure on Postprandial Metabolism in Patients with Non-Insulin-Dependent Diabetes Mellitus," American Journal of Clinical Nutrition, Vol. 61, No. 4, 1995, pp. 837-842.

[8] H. W. Heaton, S. W. Marcus, P. M. Emmett and C. H. Bolton, "Particle Size of Wheat, Maize and Oat Test Meals: Effects on Plasma Glucose and Insulin Responses and on the Rate of Starch Digestion in Vitro," American Journal of Clinical Nutrition, Vol. 47, No. 4, 1988, pp. 675-682.

[9] K. S. Juntunen, L. K. Niskanen, K. H. Liukkonen, K. S. Poutanen, J. J. Holst and H. M. Mykkanen, "Postprandial 
Glucose, Insulin, and Incretin Responses to Grain Products in Healthy Subjects," American Journal of Clinical Nutrition, Vol. 75, No. 2, 2002, pp. 254-262.

[10] K. O’Dea, P. J. Nestel and L. Antonoff, "Physical Factors Influencing Postprandial Glucose and Insulin Responses to Starch," American Journal of Clinical Nutrition, Vol. 33, No. 4, 1980, pp. 760-765.

[11] J. Hallfrisch and K. M. Behall, "Mechanism of Effects of Grain on Insulin and Glucose Responses," Journal of American College of Nutrition, Vol. 19, No. 3, 2007, pp. 320S-325S.

[12] M. J. Throne, L. U. Thompson and D. J. A. Jenkins, "Factors Affecting Starch Digestibility and the Glycemic Re- sponse with Special Reference to Legumes," American Journal of Clinical Nutrition, Vol. 38, No. 3, 1983, pp. 481-488.

[13] T. M. S. Wolever, "Dietary Carbohydrates and Insulin Action in Humans," British Journal of Nutrition, Vol. 83, Suppl. 1, 2000, pp. 97-102.

[14] S. H. A. Holt, J. C. B. Miller and P. Petocz, "An Insulin Index of Foods: Insulin Demand Generated by $1000 \mathrm{~kJ}$ Portions of Common Food," American Journal of Clinical Nutrition, Vol. 66, No. 5, 1997, pp. 1264-1276. 